greater time spent immobile. Both groups increased their daily activity count and decreased their immobile time between discharge and follow-up. However, those with preserved RFcsa tended to increase their activity count more than those with RFcsa wasting (table 1).

Discussion These data suggest RFcsa muscle wasting as a consequence of an AECOPD was more common in older patients with a lower BMI. These patients tended to have a longer hospital stay with lower activity levels both during and immediately after an AECOPD However, more patients are required to confirm these initial observations.

Abstract P40 Table 1 Differences in physical activity levels between group 1 and group 2

\begin{tabular}{|c|c|c|}
\hline & $\begin{array}{l}\text { Group } 1 \\
\text { RFcsa } \geq 10 \% \text { loss } \\
\text { Mean } \pm \text { SD }\end{array}$ & $\begin{array}{l}\text { Group } 2 \\
\text { RFcsa }<10 \% \text { loss } \\
\text { Mean } \pm \text { SD }\end{array}$ \\
\hline \multicolumn{3}{|l|}{ DEMOGRAPHICS } \\
\hline Age (years) & $75.8 \pm 13.1$ & $66.2 \pm 7.5$ \\
\hline BMI $\left(\mathrm{kg} / \mathrm{m}^{2}\right)$ & $21.8 \pm 7.6$ & $30.2 \pm 8.7$ \\
\hline Length of stay (days) & $10.8 \pm 10.3$ & $4.0 \pm 4.5$ \\
\hline $\begin{array}{l}\text { Admission Numerical Rating Scale for } \\
\text { dyspnoea (0-10) }\end{array}$ & $8.3 \pm 0.5$ & $4.6 \pm 2.7$ \\
\hline Admission COPD Assessment Tool (0-40) & $32 \pm 4$ & $26 \pm 8$ \\
\hline $\mathrm{FEV}_{1}(\mathrm{~L})$ & $0.7 \pm 0.14$ & $0.6 \pm 0.09$ \\
\hline Discharge Numerical Rating Scale $(0-10)$ & $5.4 \pm 3.2$ & $2.2 \pm 1.8$ \\
\hline Discharge COPD Assessment Tool (0-40) & $25 \pm 6$ & $19 \pm 12$ \\
\hline \multicolumn{3}{|l|}{ ACTIVITY } \\
\hline Activity count at discharge (AU) & $87,699 \pm 52,786$ & $169,548 \pm 92,657$ \\
\hline Activity count/minute at discharge (AU/min) & $93 \pm 52$ & $185 \pm 88$ \\
\hline Immobile time at discharge (minutes) & $254 \pm 111$ & $169 \pm 153$ \\
\hline Percentage immobile time (\%) & $28 \pm 13$ & $18 \pm 14$ \\
\hline Activity count at 4 week follow up (AU) & $148,073 \pm 87,759$ & $213,810 \pm 65,438$ \\
\hline $\begin{array}{l}\text { Activity count/minute at } 4 \text { week follow up } \\
\text { (AU/min) }\end{array}$ & $159 \pm 85$ & $238 \pm 68$ \\
\hline Immobile time at 4 week follow up (minutes) & $216 \pm 124$ & $123 \pm 59$ \\
\hline $\begin{array}{l}\text { Percentage immobile time at } 4 \text { week follow } \\
\text { up (\%) }\end{array}$ & $23 \pm 12$ & $14 \pm 7$ \\
\hline
\end{tabular}

$\mathrm{BMI}=$ Body Mass Index, RFcsa = Rectus Femoris cross-sectional area, $\mathrm{AU}=$ Arbitrary units.

\section{P41 THE ROLE OF OBESITY IN UNEXPLAINED BREATHLESSNESS AND EXERCISE INTOLERANCE EVALUATED BY CARDIO- PULMONARY EXERCISE TESTS (CPET)}

doi:10.1136/thoraxjnl-2012-202678.182

P James, Z Mangera, S Isse, B Ibrahim, R Gupta, K Wadsworth, D Mukherjee, B Yung, JT Samuel. Basildon and Thurrock University Hospital, Basildon, United Kingdom

Introduction Resting cardio-respiratory investigations may not indicate a cause for unexplained breathlessness and exercise intolerance (UB\&EI). CPET is an objective, non-invasive tool to measure cardio-respiratory function during exercise. Increased BMI can contribute to exercise intolerance. We present a review of CPET in evaluating patients referred to our centre with UB\&EI.

Methods We did retrospective analysis of CPET results in patients referred to our centre over a two year period (2010 to 2012). All patients had resting cardio-respiratory function tests including coronary angiogram and stress echo cardiogram in some cases. CPET was performed by maximum symptom limited incremental protocol on a cycle ergometer starting with 3 minutes of rest followed by 3 minutes of unloaded cycling and subsequent increase in workload as per ATS recommendations. We defined obesity as BMI of $\geq 30 \mathrm{~kg} /$ $\mathrm{m}^{2}$. Tests were supervised by two qualified physiologists and reported by a consultant chest physician.
Results Of 243 CPET tests performed during this period, 68 (28\%) were done to evaluate UB\&EI. 38 patients $(56 \%)$ were obese. Overall, obesity was the sole cause of UB\&EI in 22 patients (32\%) and was a contributory factor in a further 15 patients $(22 \%)$. In the obese subgroup, it was the sole cause in 21 patients (55\%) and contributory in a further 16 patients (43\%). Being overweight (BMI 29) was the sole cause of unexplained breathlessness in 1 non-obese patient. Obesity appeared to be the major contributing factor for UB\&EI even when present with other factors. In 23 patients $(34 \%)$ more than one contributory factor was present (Cardiovascular/ Respiratory/VO mismatch/physical de-conditioning/functional). Physical de-conditioning was the sole cause in 5 patients (7\%) and Hyperventilation in 2 patients (3\%).

Conclusion While the causes of UB\&EI are multi factorial, in our study obesity was a contributory and often key factor in over half the patients. In the obese subgroup, being overweight was the sole factor in over half and played a contributory role in almost all patients. CPET is a useful test to determine specific causes of UB\&EI when resting cardio-respiratory tests are non-contributory and can help with reassurance, dietary advice and exercise prescription.

\section{P42 COMPARISON OF VENTILATION HETEROGENEITY INDICES DERIVED FROM MULTIPLE BREATH INERT GAS WASHOUT}

doi:10.1136/thoraxjnl-2012-202678.183

'S Gonem, 'S Natarajan, 'S Corkill, 'A Singapuri, 'D Desai, ${ }^{2} \mathrm{P}$ Gustafsson, 'CE Brightling, 'S Siddiqui. ' ${ }^{1}$ Genfield Hospital, Leicester, United Kingdom; ${ }^{2}$ Central Hospital, Skövde, Sweden

Introduction and objectives Multiple breath inert gas washout $(\mathrm{MBW})$ is a technique for quantifying ventilation heterogeneity (VH). Commonly-used indices of VH such as lung clearance index (LCI) may be biased by alterations in functional residual capacity, tidal volume and anatomical dead space. We hypothesised that a novel marker of $\mathrm{VH}$, exponential decay index (EDI) would exhibit more favourable measurement properties than established indices. Methods Seventy-four patients with asthma and eighteen healthy control subjects were recruited. MBW was performed in triplicate in each subject using a modified Innocor gas analyser (Innovision A/S, Odense, Denmark). Analysis of the washout curve yielded a number of $\mathrm{VH}$ parameters including LCI, mixing ratio (MiR), moment ratio (MoR) and the novel parameter EDI. EDI was calculated by fitting the washout data to both one-phase and two-phase exponential decay models and deriving the difference in goodness of fit $\left(R^{2}\right)$ between the two models. Phase III slope analysis yielded values for $\mathrm{S}_{\text {cond }}, \mathrm{S}_{\text {acin }}$ and first breath SnIII (FB SnIII). Repeatability was assessed using intraclass correlation coefficient (ICC), and ability to discriminate between health and disease was determined using receiver operating characteristic (ROC) curves. Robustness to variations in FRC, $\mathrm{Vt}$ and $\mathrm{Vd}$ was determined by calculating the ratio of signal (absolute difference between healthy and asthma groups) to noise (standard deviation of calculated 'ideal' values for each subject, assuming perfect gas mixing).

Results The most repeatable parameters were LCI (ICC $=0.905)$, MoR (ICC $=0.869), F B$ SnIII $(I C C=0.867)$ and $S_{\text {acin }}($ ICC $=0.846)$. The most discriminatory parameters were FB SnIII, $\mathrm{S}_{\text {acin }}$ and EDI, with areas under the ROC curve of $0.728,0.707$ and 0.691 respectively. Signal-to-noise ratios for LCI, MiR and MoR were 1.726, 3.911 and 0.679 respectively, thus accounting for the poor discriminatory ability of these markers.

Conclusion FB SnIII and $S_{\text {acin }}$ appear to be the most favourable markers of $\mathrm{VH}$. EDI is the most discriminatory parameter that does not rely on phase III slope analysis. Since FB SnIII may be derived from a single-breath manoeuvre, this parameter warrants further investigation in other disease groups, and normative values should be derived in large healthy populations. 